\title{
Role of the MicroRNA-17-92 Cluster in the Endothelial Differentiation of Stem Cells
}

\author{
Karine Tréguer Eva-Marie Heinrich Kisho Ohtani Angelika Bonauer \\ Stefanie Dimmeler \\ Institute for Cardiovascular Regeneration, Center of Molecular Medicine, University of Frankfurt, \\ Frankfurt, Germany
}

\section{Key Words}

MicroRNAs $\cdot$ Endothelial cells $\cdot$ Stem cells

\begin{abstract}
MicroRNAs (miRs) are small non-coding RNAs that recently emerged as potent regulators of gene expression. The members of the miR-17-92 cluster have been shown to control endothelial cell functions and neovascularization; however, the regulation and function of the cluster in endothelial cell lineage commitment has not been explored. This project aimed to test the role of the miR-17-92 cluster during endothelial differentiation. We demonstrate that miR-17, miR-18, miR-19 and miR-20 are increased upon the induction of endothelial cell differentiation of murine embryonic stem cells or induced pluripotent stem cells. In contrast, miR-92a and the primary miR-17-92 transcript were downregulated. The inhibition of each individual miR of the cluster by cholesterol-modified antagomirs did not affect endothelial marker gene expression. Moreover, the combination of all antagomirs had no effect. These findings illustrate that although the miR-17-92 cluster regulates vascular integrity and angiogenesis, none of the members has a significant impact on the endothelial differentiation of pluripotent stem cells.

Copyright $\odot 2012$ S. Karger AG, Basel
\end{abstract}

\section{Introduction}

MicroRNAs (miRs) are small RNA molecules of about $21 \mathrm{nt}$ which regulate gene expression by repressing target genes at the post-transcriptional level. MiRs have unique expression profiles in different tissues and regulate many biological processes. Several of them control endothelial cell functions and vessel growth after induction of ischemia (for review see $[1,2]$ ). Among them, the miR-17-92 cluster has been shown to play a crucial role in angiogenesis [3]. This cluster is a polycistronic miR gene, encoding 7 mature miRs, namely miR-17-3p, miR-17-5p, miR-18a, miR-19a, miR-20a, miR-19b and miR-92a, all of which are processed from a single primary transcript [4]. Based on the seed sequence composition, these miRs can be grouped into 4 families: MiR-17/20, miR-18, miR-19 and miR-92a. The miR-17-92 paralog clusters miR-106b-25 and miR-106a-363 were generated by gene duplication representing homologous miRs, thereby adding more complexity and redundancy in mammalian cells $[5,6]$.

First insights into the function of the miR-17-92 cluster in angiogenesis came from studies in which this cluster

K.T. and E.-M.H. contributed equally to this paper.

\section{KARGER}

Fax +4161306 1234

E-Mail karger@karger.ch

www.karger.com (c) 2012 S. Karger AG, Basel

1018-1172/12/0495-0447\$38.00/0

Accessible online at:

www.karger.com/jvr
Dr. Stefanie Dimmeler

Department of Internal Medicine III, Institute for Cardiovascular Regeneration University of Frankfurt, Theodor-Stern-Kai 7

DE-60590 Frankfurt (Germany)

Tel. +496963015158 or 6667, E-Mail Dimmeler@em.uni-frankfurt.de 
was overexpressed in tumor cells. The miR overexpressing tumor cells were shown to increase tumor angiogenesis and growth, whereas tumor cell proliferation was not directly affected. This proangiogenic effect has been attributed to the regulation of antiangiogenic growth factors such as connective tissue growth factor (CTGF) and thrombospondin, which are controlled in tumor cells by miR-18 and miR-19, respectively [7]. When addressing the cell intrinsic function of the miR-17-92 cluster in endothelial cells, several members were shown to exhibit antiangiogenic effects when being overexpressed in the cells. Specifically, miR-92a profoundly inhibits angiogenesis and disturbs vessel patterning in zebrafish, in part by repression of the target gene integrin $\alpha 5$, whereas antagomir-based inhibition of miR-92a in mice augmented neovascularization and functional recovery after ischemia [8]. MiR-17 and its closely related family member miR-20 also reduced sprouting angiogenesis in vitro and in vivo, but increased proliferation [9]. The combination of miR-17, miR-18 and miR-20 partially rescued the defective proliferative response in Dicer-deficient endothelial cells [10]. The stimulation of angiogenesis and new blood vessel growth is a therapeutic option to treat patients suffering from ischemic diseases. The growth of blood vessels is tightly controlled and it has been shown that various stem or progenitor cell subsets as well as proangiogenic myeloid cells can contribute to neovascularization after ischemia $[11,12]$. However, the mechanisms underlying the differentiation of endothelial cells and the involvement of miRs in this process are unclear. Recently, several miRs were shown to be regulated during the endothelial differentiation of mouse or human embryonic stem cells (ESCs) [13]. MiR-126, which is encoded by the EGFL7 gene, is upregulated during growth-factor-enhanced endothelial differentiation [14] and is enriched in sorted CD31+ cells isolated from ESC-derived embryoid bodies [15]. Moreover, several proangiogenic miRs such as miR-130a, miR-210 and members of the let-7 family were increased upon the induction of endothelial differentiation [14]. The miR-17-92 cluster members miR-17, miR18a, miR-19a/b and miR-20a were among the most highly enriched miRs in ESCs in some but not all studies [16].

In this study, we specifically determined the regulation of the miR-17-92 cluster during endothelial differentiation in mESCs. Since induced pluripotent stem cells, generated by the overexpression of pluripotency-inducing genes in fibroblasts [17], are an alternative tool to study cell differentiation, we also compared the regulation and function of the miR-17-92 cluster in both mESCs and mouse induced pluripotent stem cells (miPSCs).

\section{Material and Methods}

\section{mESCs Culture}

Mouse ESCs (R1/E, SCRC 1036) were cultured without feeder cells on gelatin-coated dishes in DMEM supplemented with 15\% FBS, $150 \mu \mathrm{M}$ monothioglycerol (Sigma), $0.1 \mathrm{mM}$ nonessential amino acids (Gibco), penicillin-streptomycin and 1,000 U/ml leukemia inhibitory factor (LIF, StemCell Technology).

\section{miPSCs Culture}

Stemgent mouse primary iPS cells-WP5 were maintained on mouse embryonic fibroblasts (MEFs; Global Stem, Rockville, Md., USA) in miPSC growth medium (knockout D-MEM, 14\% FBS, nonessential amino acids, $1.85 \mathrm{~mm}$ L-glutamine, penicillinstreptomycin, $0.05 \mathrm{mM} \beta$-mercaptoethanol, $463 \mathrm{U} / \mathrm{ml} \mathrm{LIF}$ ).

\section{Endothelial Differentiation}

Differentiation of mESCs or miPSCs into embryoid bodies was performed using the suspension method as previously published $[18,19]$. Cells $\left(1.25 \times 10^{5}\right)$ were seeded in $10 \mathrm{ml}$ of differentiation medium which included either a cytokine cocktail or vascular endothelial growth factor (VEGF). Specifically, we used two different protocols to induce endothelial differentiation. (1) For the cytokine cocktail-protocol, the cells were kept for 7-11 days in differentiation medium [IMDM Glutamax (Gibco) supplemented with 15\% FBS, $450 \mu \mathrm{M}$ monothioglycerol, penicillinstreptomycin, $10 \mu \mathrm{g} / \mathrm{ml}$ insulin, $50 \mathrm{ng} / \mathrm{ml} \mathrm{hVEGF,} 2 \mathrm{U} / \mathrm{ml}$ human erythropoietin (Epo), $100 \mathrm{ng} / \mathrm{ml}$ hbFGF, $10 \mathrm{ng} / \mathrm{ml} \mathrm{IL-6].} \mathrm{(2)} \mathrm{For}$ the VEGF-protocol, the mESC maintenance medium without LIF was used. The cells were cultivated for 2 days on nonadhesive 100 $\mathrm{mm}$ dishes. After 2 days, $30 \mathrm{ng} / \mathrm{ml}$ of hVEGF121 (PeproTech) was added. The cells were further cultivated and harvested after 4, 7 and 11 days of differentiation for analysis.

\section{Spheroid Culture}

After 8 days of differentiation, mESCs and miPSCs were dissociated into a single cell suspension using a solution of trypsin and EDTA for $2 \mathrm{~min}$ at $37^{\circ} \mathrm{C}$. The spheroid assay was then performed using the previously described protocol $[20,21]$ in the cytokine medium: $4.8 \times 10^{4}$ cells were mixed with $12 \mathrm{ml}$ of a mixture containing $80 \%$ differentiation medium and $20 \%$ methocoel (BD). This cell suspension was dispensed into the wells of a 96well plate with nonadhesive U-bottoms (100 $\mu$ l per well). Thereafter, the plate was incubated at $37^{\circ} \mathrm{C}$ for $24 \mathrm{~h}$ to allow spheroid formation. The next day the spheroids were harvested and overlayed with a mixture of $80 \%$ methocoel and $20 \%$ FCS. The collagen gel was prepared $(500 \mu \mathrm{l}$ of $10 \times$ M199 media (Sigma), $4 \mathrm{ml}$ of collagen stock solution (rat-tail collagen type I, BD), $90 \mu \mathrm{l}$ of $1 \mathrm{M}$ HEPES, $400-800 \mu \mathrm{l} \mathrm{NaOH}$ and $1 \mathrm{ml}$ of penicillin/streptomycin) and mixed with the spheroid suspension in a 1:1 volume ratio. On a 24 -well plate, $1 \mathrm{ml}$ of this mixture was plated per well, followed by incubation at $37^{\circ} \mathrm{C}$. After solidification, the gels were overlayed with $100 \mu \mathrm{l}$ of differentiation medium (cytokine medium) with or without the addition of hVEGF (50 ng/ml) and then incubated for 9 days.

\section{Antagomir Treatment}

Antagomirs were designed and synthesized by VBC Biotech, Vienna, as previously described [9]. They had the following sequences: Antagomir-17: 5'-CUACCUGCACUGUAAGCACUU- 
UG-3'; Antagomir-18a: 5'-CUAUCUGCACUAGAUGCACCUUA-3'; Antagomir-19a: 5'-UCAGUUUUGCAUAGAUUUGCACA-3'; Antagomir-20a: 5'-CUACCUGCACUAUAAGCACUUUA-3'; Antagomir-92a:3'-CAGGCCGGGACAAGUGCAAUA-5'; Antagomir-Co: 5'-AAGGCAAGCUGACCCUGAAGUU-3' and were added at the first day of differentiation at a final concentration of $150 \mathrm{nM}$.

\section{Quantification of miRNAs and Gene Expression Using} Quantitative Real-Time PCR

To analyze miR expression by quantitative real-time PCR (qRT-PCR), 10 ng of RNA were reverse-transcribed using miRspecific primers from Applied Biosystems (TaqMan Assays; online suppl. tables; for all online suppl. material, see www. karger.com/doi/10.1159/000339429). qRT-PCR was performed using $1.4 \mu \mathrm{l}$ of samples and snRNA U6 as an internal control.

For quantification of mRNA expression, first-strand synthesis was performed on $1 \mu \mathrm{g}$ of RNA using MLVRT (Applied Biosystems, Carlsbad, Calif., USA) in a $20-\mu l$ reaction. Two different technologies were used, SYBR green (Applied Biosystems, Darmstadt, Germany) and TaqMan. With the SYBR green method, $\beta$-actin was used as an internal control (online suppl. table 1). TaqMan gene expression assays were purchased from Applied Biosystems and PolR2A was used as an internal control (online suppl. table 2). After diluting to a final volume of $200 \mu \mathrm{l}$, $5 \mu \mathrm{l}$ of samples were used in duplicate for qRT-PCR with an ABI StepOne Plus real-time PCR thermocyler. All primer sets for mRNAs crossed an exon-exon junction to avoid the amplification of genomic DNA. SYBR green was used for the analysis of Oct4, Nanog, Brachyury, VE-Cadherin, Tal1, Runx1 and smooth muscle actin (SMA). TaqMan assays were used for the expression of Tie2 and platelet endothelial cell adhesion molecule (PECAM).

\section{Stainings}

To stain the differentiated cells, 4,000 cells per chamber were seeded at day 0 on 4 chamber glass slides (Nunc-177399). After 7 days of cultivation in the absence of LIF, and the addition of 30 $\mathrm{ng} / \mathrm{ml}$ hVEGF at day 2, cells were fixed in $2 \%$ PFA, blocked in $\mathrm{PBS} / 1 \% \mathrm{BSA}$ (for vWF staining) or HEPES/1\% BSA (for the IsoLectin B4 staining), for $20 \mathrm{~min}$ at room temperature. The antibody directed against vWF (Acris) was used 1:100 in the blocking buffer, and Iso-Lectin BA (Vector B1205) 1:200 in its blocking buffer for $1 \mathrm{~h}$ at room temperature. A secondary antibody was used for the Iso-Lectin B4 staining with 1:200 streptavidin Alexa 555 for $1 \mathrm{~h}$ at room temperature. For the acetylated LDL uptake assay, $10 \mu \mathrm{g} / \mathrm{ml}$ acetylated LDL (Life Technologies) was added to the medium of the mESCs, incubated for $4 \mathrm{~h}$ at $37^{\circ} \mathrm{C}$ and washed 3 times with PBS. The slides were mounted in 1:500 Hoechst mounting-medium and images were obtained by the fluorescent microscopy and confocal microscope (Zeiss) (LSM).

\section{Statistical Analysis}

Statistical analysis was performed with the GraphPad Prism5.3 program. For multiple comparisons, nonparametric 1-way ANOVA analysis with a Dunn's posttest (comparing all pairs of columns) was performed. Data are shown as a mean and standard error of the mean.

\section{Results}

\section{Induction of Endothelial Differentiation in mESCs} and miPSCs

In order to determine the regulation of miRs during endothelial differentiation, we used two different published protocols and analyzed the expression of pluripotency genes and mesodermal and endothelial markers over time. The first protocol [22] uses the differentiation medium including a cytokine cocktail (see Methods: 'cytokine cocktail-protocol'). When using this protocol, the expression of the pluripotency genes Oct4 and Nanog declined within days, whereas the mesoderm marker Brachyury was transiently increased at day 4 . In accordance with these data, the mesoderm miR miR-302b [23] was also transiently upregulated at day 4 (fig. 1).

Consistent with previous publications [15], some endothelial markers such as CD31 (PECAM) or Tie2 were expressed in undifferentiated mESCs and miPSCs. Upon induction of endothelial differentiation, the expression of PECAM and Tie2 was downregulated at day 4 and then re-expressed at day 7. On the other hand, VE-cadherin, a prototypical marker of mature endothelial cells, was not expressed in undifferentiated cells and showed a gradual increase along with the differentiation which peaked at day 7 (fig. 2). No further augmentation of endothelial marker gene expression was seen for up to 15 days of observation (data not shown). Consistent with the upregulation of endothelial markers, the endothelial-enriched miR-126 [15, 24] was increased upon differentiation. Worthy of note: the expression pattern of the markers was similar in mESCs and miPSCs (fig. 2).

In a second protocol, endothelial differentiation was initiated by the removal of LIF and the addition of VEGF [25] (see Methods: 'VEGF protocol'). The time course and the expression pattern of pluripotency genes and mesodermal and endothelial markers were similar compared to the results obtained with the 'cytokine-protocol' using VEGF, Epo, bFGF and IL-6 (online suppl. fig. 1). Comparison of the two different protocols revealed that the VEGF protocol more efficiently induced differentiation compared to the cytokine protocol (online suppl. fig. 1).

To determine the morphology of the differentiated cells and the protein expression of pluripotency and endothelial markers, we also performed immunostainings. As expected, undifferentiated mESCs were organized in colonies and expressed high levels of nuclear localized Oct4 (data not shown). After 7 days of endothelial differentiation, the cells showed a cobblestone-like morphology and expressed von Willebrand factor (fig. 3a) and 


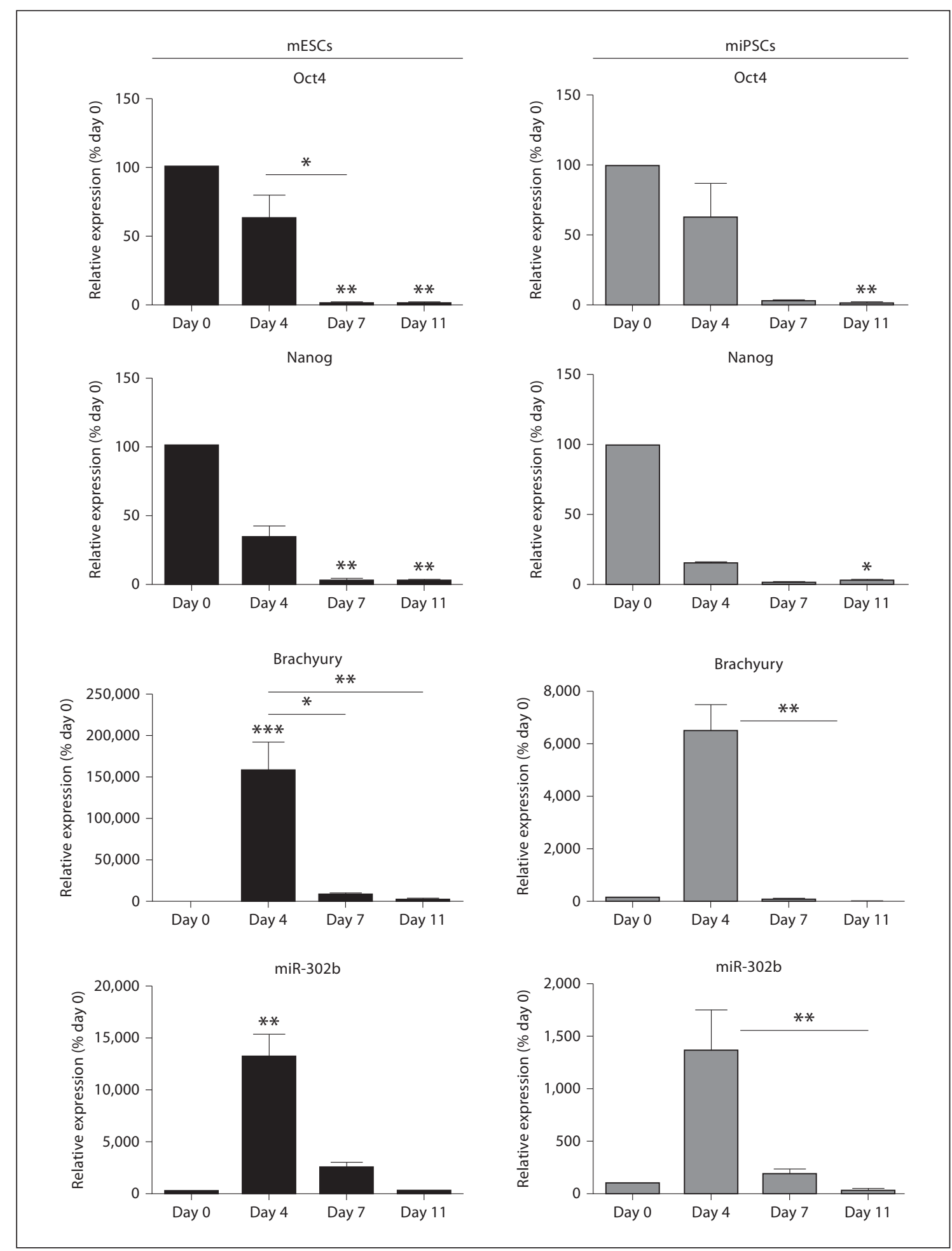

Fig. 1. Expression of pluripotency and mesodermal markers during endothelial differentiation of mESCs and miPSCs. mESCs and miPSCs were kept for 11 days in endothelial differentiation medium containing Epo, bFGF, VEGF and IL-6 (cytokine protocol). Differentiation was stopped at days $0,4,7$ and 11 . The gene expression was analyzed via qRT-PCR. The data were normalized against $\beta$-actin (Oct4, Nanog, Brachyury) or snRNA U6 (miR$302 \mathrm{~b})$ and are depicted as percentage of day 0 (= undifferentiated cells). mESCs: $\mathrm{n} \geq 7$, miPSCs: Oct4, Nanog, miR-302b, $\mathrm{n}=3$; Brachyury: $n=6$. The data are presented as mean \pm SEM (standard error of the mean). ${ }^{*} \mathrm{p}<0.05,{ }^{* *} \mathrm{p}<0.01,{ }^{* * *} \mathrm{p}<0.001$. 


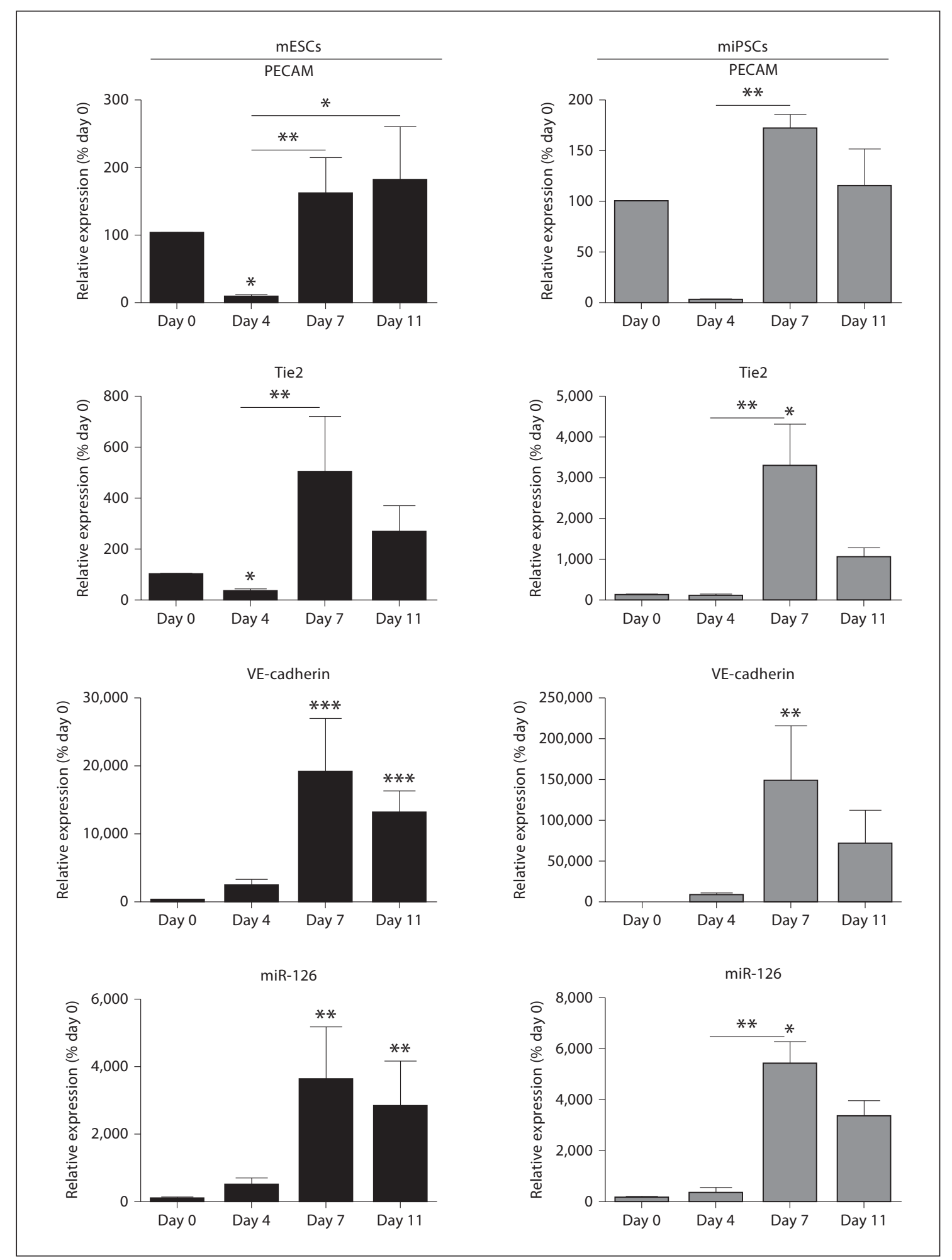

Fig. 2. Expression of endothelial markers during endothelial differentiation of mESCs and miPSCs. mESCs and miPSCs were kept in endothelial differentiation medium (cytokine protocol) for 11 days. The gene expression was analyzed at the indicated time points by qRT-PCR. The data were normalized against $\beta$ - actin (VE-cadherin), PolR2A (Tie2 and PECAM) or snRNA U6 (miR-126) and are depicted as percentage of day 0 (= undifferentiated cells). mESCs: $\mathrm{n} \geq 7$, miPSCs: $\mathrm{n} \geq 5$. The data are presented as mean \pm SEM. ${ }^{*} \mathrm{p}<0.05,{ }^{* *} \mathrm{p}<0.01,{ }^{* *} \mathrm{p}<0.001$. 

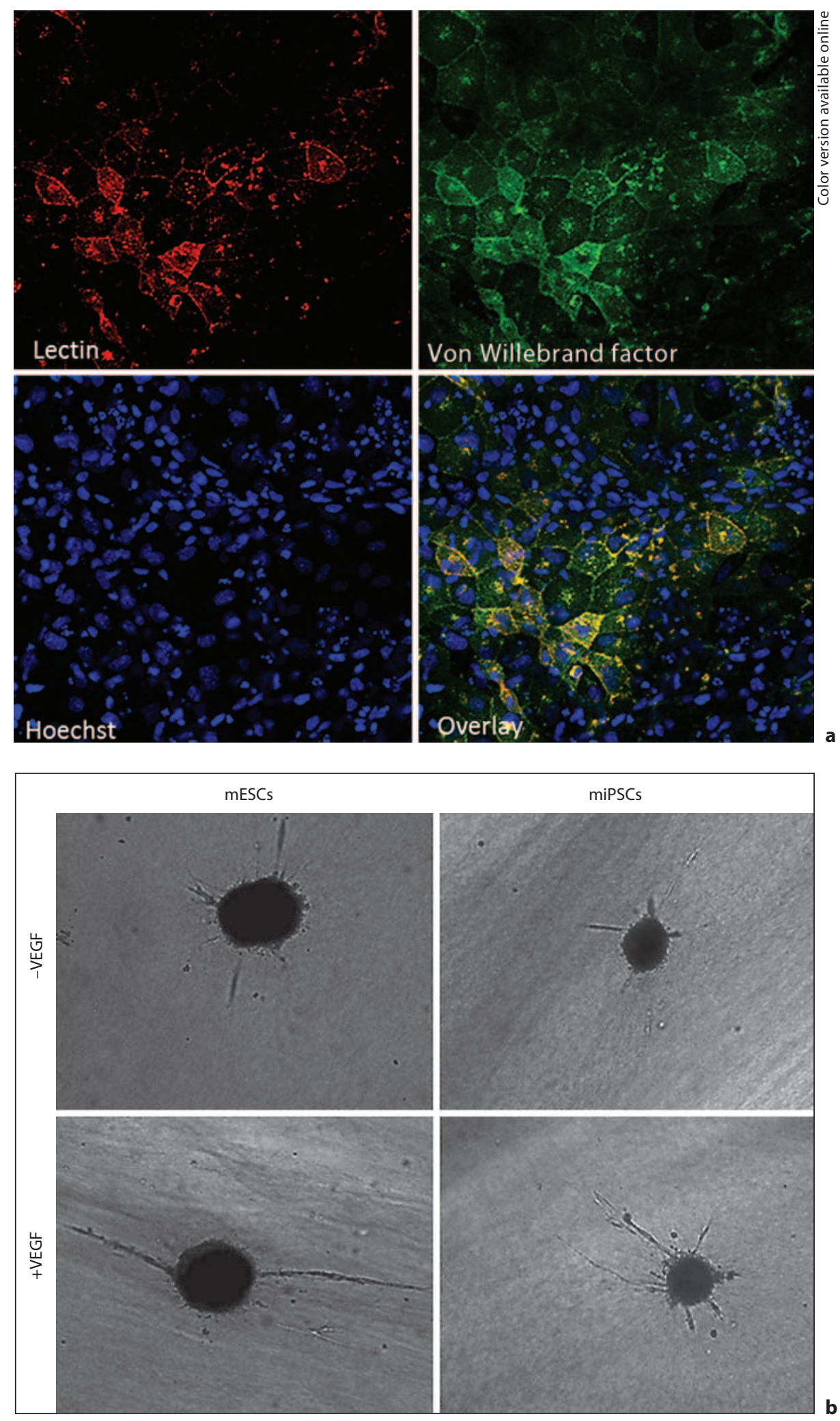

Fig. 3. Endothelial marker gene expression in differentiated mESCs. a mESCs were analyzed by immunostaining on chamber slides. Differentiated cells were stained for Iso-Lectin B4 (red) and von Willebrand factor (green). Hoechst dye was used for nuclear staining (blue). b After 8 days of differentiation of mESCs and miPSCs, the spheroid assay was started with endothelial differentiation medium according to the cytokine protocol. The cells were kept with or without addition of VEGF (50 ng/ $\mathrm{ml})$. Spheroids were observed daily and images were taken 17 days after starting the differentiation protocol. 
CD31 (data not shown), bound Iso-Lectin B4 (fig. 3a) and incorporated DIL-labeled acetylated LDL (data not shown). In contrast, the pluripotency marker Oct4 was downregulated in these differentiated cells (data not shown).

Having demonstrated that, under the differentiation conditions used, the cells were losing pluripotency markers while gaining endothelial markers, the functionality of the cells was tested. Therefore, a spheroid assay was performed to show that the differentiated stem cells can recapitulate sprouting angiogenesis [18]. The spheroids were kept with or without VEGF stimulation. As shown in figure $3 \mathrm{~b}$, the spheroids made of mESCs as well as miPSCs formed sprouts, which were further increased in the presence of VEGF, documenting the functional activity of the differentiated stem cells.

\section{Expression of the miR-17-92 Cluster during}

\section{Endothelial Differentiation}

Using the established and reproducible protocols, we further analyzed miR-17-92 expression and function during endothelial differentiation in mESCs and miPSCs. All members were expressed in undifferentiated cells; however, their expression was differentially regulated during the induction of endothelial differentiation. MiR-17, miR-18, miR-19 and miR-20 were increased during endothelial differentiation with a maximal level reached at day 7 in both mESCs and miPSCs (fig. 4). Interestingly, miR-92a was decreased during endothelial differentiation. Its expression was downregulated on day 11 to $65 \pm 8$ or $77 \pm 33 \%$ compared to undifferentiated mESCs or miPSCs, respectively. Since the individual members of the family were differentially expressed during differentiation, we also determined the expression of the primary miR-17-92 transcript. The expression of pri-miR-17-92 was downregulated during differentiation with similar kinetics as miR-92a (fig. 4).

\section{Contribution of the miR-17-92 Cluster to Endothelial Differentiation}

In order to address the contribution of the miR-17-92 cluster to endothelial differentiation, each individual miR of the cluster was inhibited by cholesterol-modified antagomirs as previously described [9]. Treatment of mESCs with $150 \mathrm{nM}$ antagomir against the members of the miR-17-92 cluster resulted in long-lasting suppression of the targeted miRs until day 7 (fig. 5). Only antagomir-17 also suppressed miR-20 while antagomir-20 reduced miR-17 expression (fig. 5), a finding that can be explained by the similar sequences of these two miRs, which differ only in two nucleotides [9]. The efficiency of the antagomir treatment was confirmed by analyses of the miR-92a targets SIRT1 and PTEN, which were increased in antagomir-92-treated cells (data not shown).

After having established the knockdown of the miRs in mESCs, we determined the influence of each antagomir on the expression of pluripotency, mesodermal, endothelial and smooth muscle marker genes. As shown in figure 6 none of the antagomirs influenced the downregulation of the pluripotency gene Oct4. Moreover, the transient increase in the mesoderm marker Brachyury was also not affected (fig. 6). Endothelial marker gene expression was increased in all groups and no obvious change in the extent or the kinetics of endothelial markers was detectable (fig. 6). Likewise, SMA expression, increased during differentiation, was not influenced by the antagomirs (fig. 6). Similar results were obtained when analyzing antagomir-treated miPSCs (data not shown). As it is believed that endothelial cells and hematopoietic cells derive from the same precursor, called a hemangioblast [26,27], we also analyzed the hematopoietic markers, Tal1 and Runx1 [28, 29]. Interestingly, antagomir-92a reduced the expression of these markers (fig. 6).

To further analyze the effect of the knockdown of these miRs on endothelial morphology, we performed a staining with Lectin. However, no difference was detectable between the antagomir-treated and control cells (fig. 7a).

Since some of the members of the cluster share the same seed sequence and may compensate for each other, we also used a cocktail of antagomirs to suppress all five miRs. As a control, we used a higher concentration of control-antagomir (control ${ }^{*} 5,750 \mathrm{nM}$ ) to exclude unspecific effects caused by a higher concentration of the antagomir mixture. However, the combined knockdown of all miRs also did not influence the kinetics or extent of marker gene expression and only the smooth muscle marker SMA and the hematopoietic markers Tall and Runx1 were modestly, but not significantly, reduced (fig. 8). Again, Lectin binding was not affected by the pooled antagomir treatment (fig. $7 \mathrm{~b}$ ). In addition, we analyzed the DIL-labeled acetylated LDL uptake and showed that it is not modified by the pooled antagomir treatment (fig. 7c). Taken together, these results indicate that the members of the miR-17-92 cluster did not significantly affect mESC pluripotency or endothelial differentiation. 
Fig. 4. Expression of the miR-17-92 cluster members during endothelial differentiation of mESCs and miPSCs. mESCs and miPSCs were differentiated for 11 days (cytokine protocol), and miR-17-92 cluster member expression was analyzed by qRT-PCR. The results are presented as relative expression, normalized to snRNA U6 (miRs) or $\beta$-actin (pri-miR-17-92) expression and depicted as percentage of day 0 (= undifferentiated cells). $\mathrm{n} \geq 3$. The data are presented as mean \pm SEM (standard error of the mean). ${ }^{*} \mathrm{p}<0.05,{ }^{* *} \mathrm{p}<0.01$, compared to day 0 .
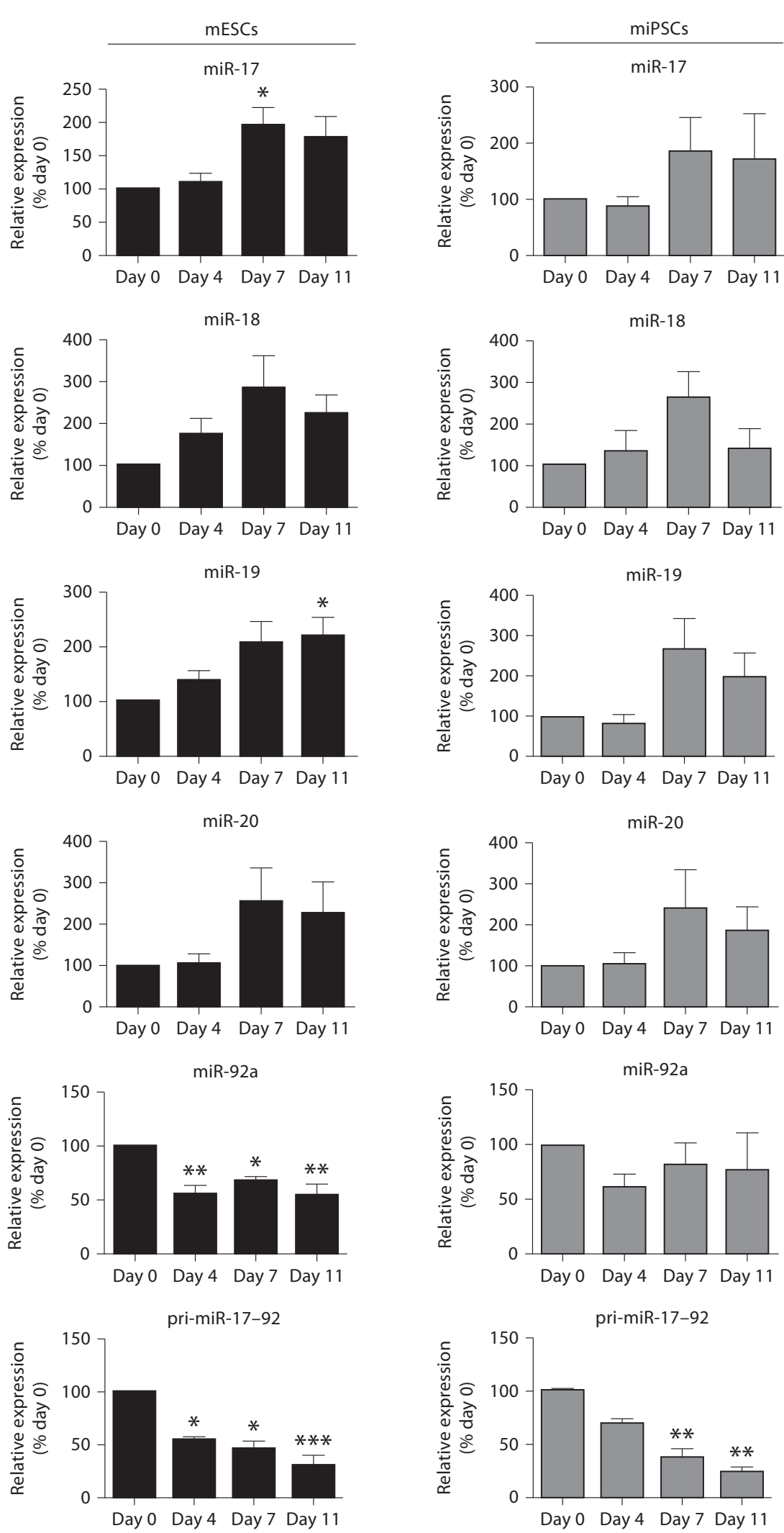
Fig. 5. Inhibition of the miR-17-92 cluster members by antagomirs in mESCs. Antagomirs $(150 \mathrm{nM})$ were added at day 0 . mESCs were differentiated for 7 days (VEGF protocol) and the expression of the miR-17-92 cluster members was detected at the respective time point by qRT-PCR. The data are depicted unnormalized as \% antagomir control. $n=2-3$. The data are presented as mean \pm SEM unless $\mathrm{n}=2$. Antag. = Antagomir; co $=$ control. ${ }^{*} \mathrm{p}<$ 0.05 compared to antagomir control.
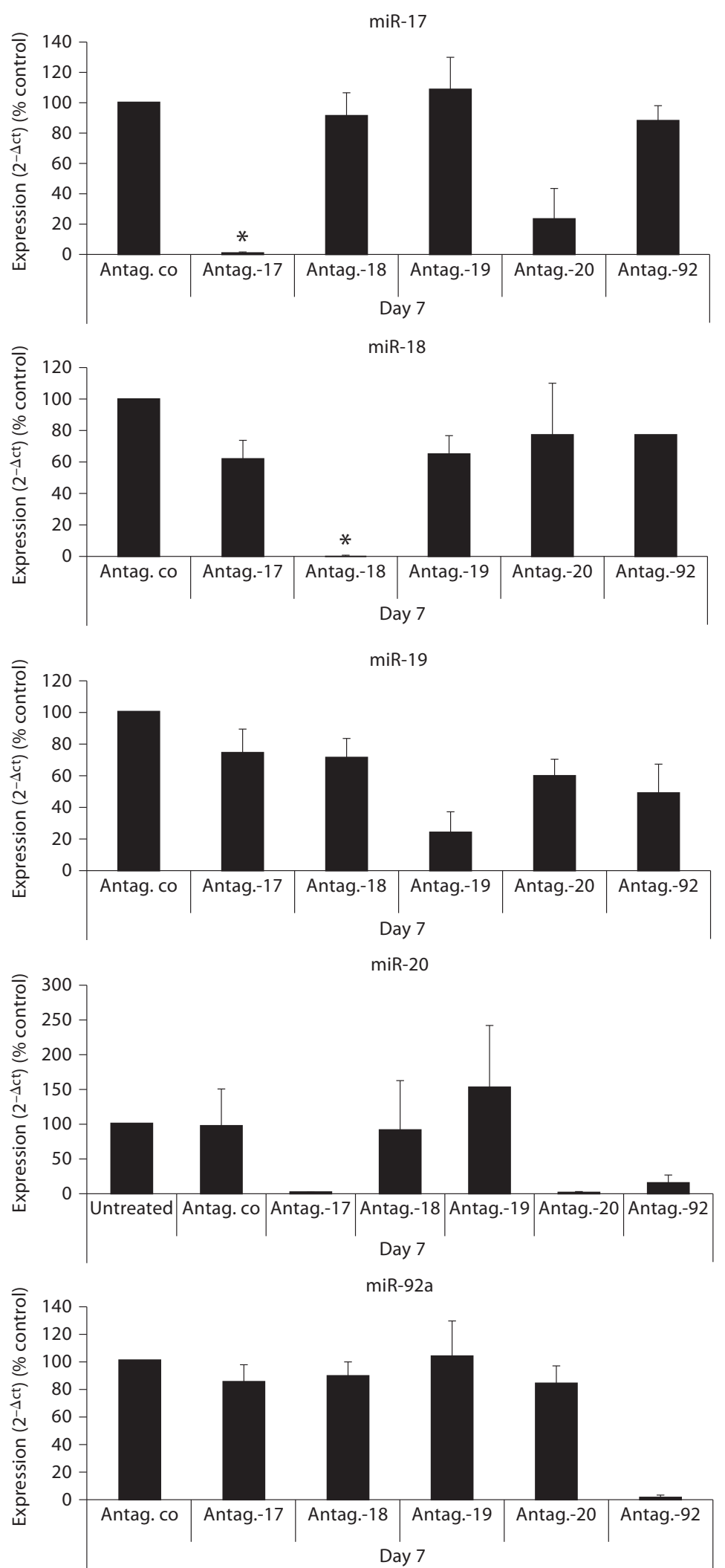


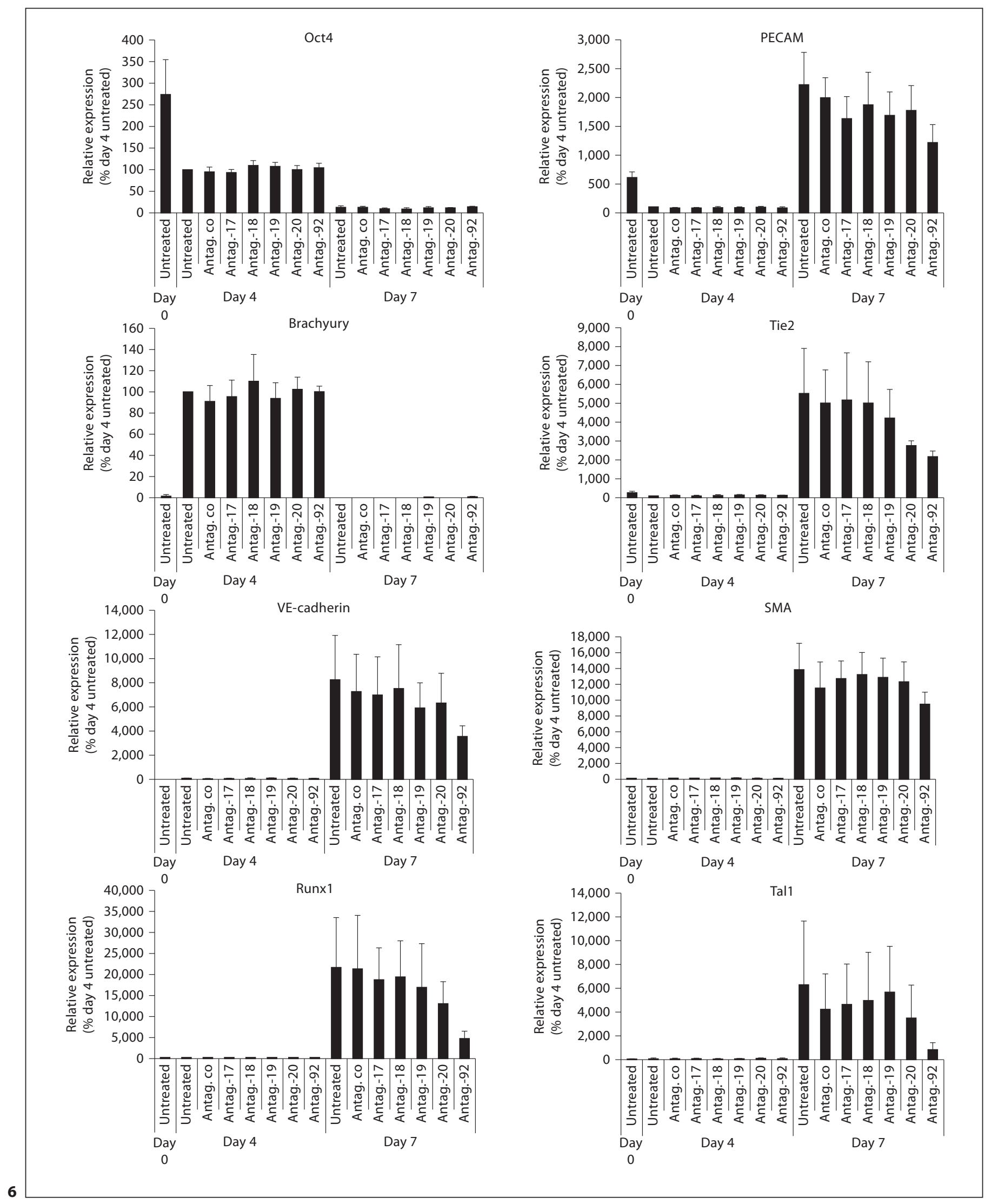


a

against the miR-17-92 cluster on marker gene expression. The experiment was carried out as described in figure 5 and the expression of the markers was determined by $\mathrm{qPCR}$. The data are normalized to $\beta$ actin (Oct4, Brachyury, VE-Cadherin, SMA, Tal1 and Runx1) or PolR2A (Tie2, PECAM) and are depicted as mean relative expression (\% untreated day 4) \pm SEM. $\mathrm{n}=3-4$. Antag. = Antagomir; $\mathrm{co}=$ control; untr. = untreated.

Fig. 7. Antagomir treatment of mESCs during endothelial differentiation does not alter the expression of Lectin or the acetylated LDL uptake. mESCs were cultivated in endothelial differentiation medium (VEGF-protocol) for 7 days. Cells were then stopped and incubated with Lectin and counterstained with Hoechst to visualize the nuclei. a Cells after 7 days of differentiation were either untreated or treated with a control antagomir or antagomirs against miR-17 or miR-92a. b Antagomir control-treated cells and cells treated with antagomirs against miR-17, miR-18, miR19, miR-20 and miR-92a. c Acetylated LDL uptake of cells treated with antagomir control or antagomirs against miR-17, miR-18, miR-19, miR-20 and miR-92a.

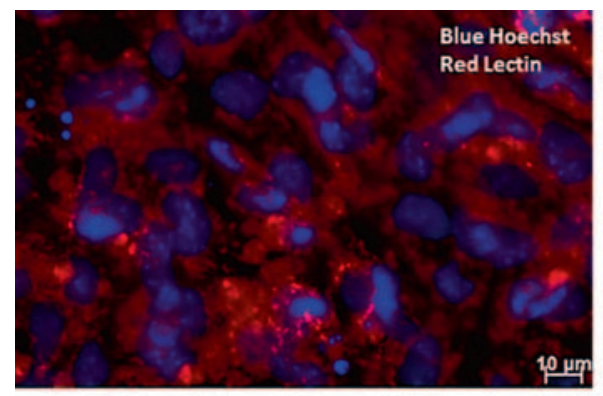

Antagomir-17
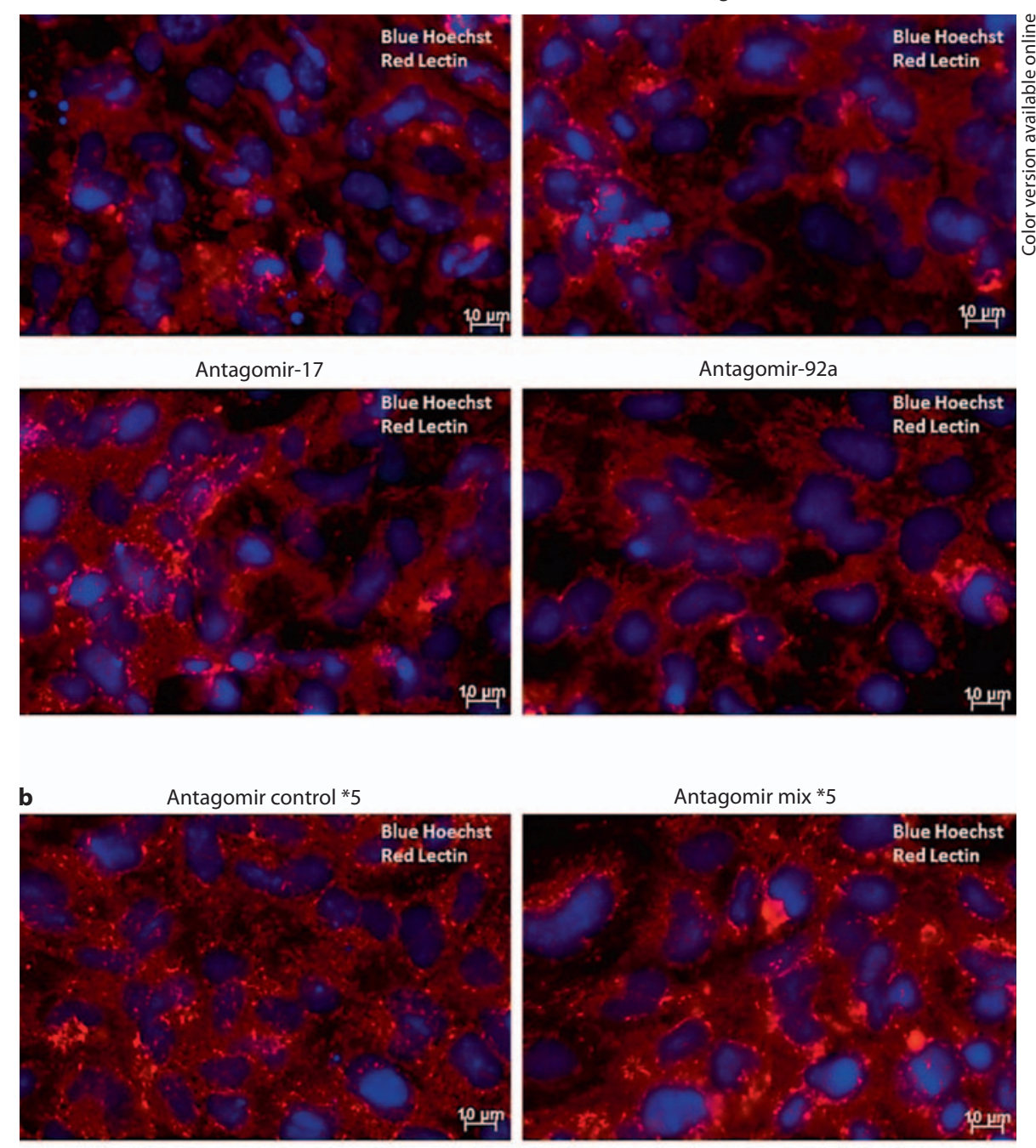

C

Antagomir control *5
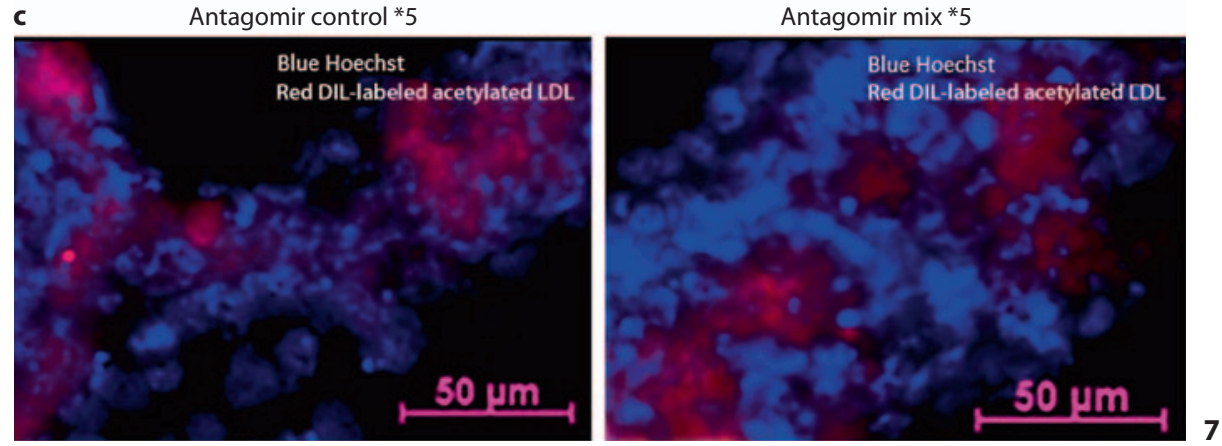


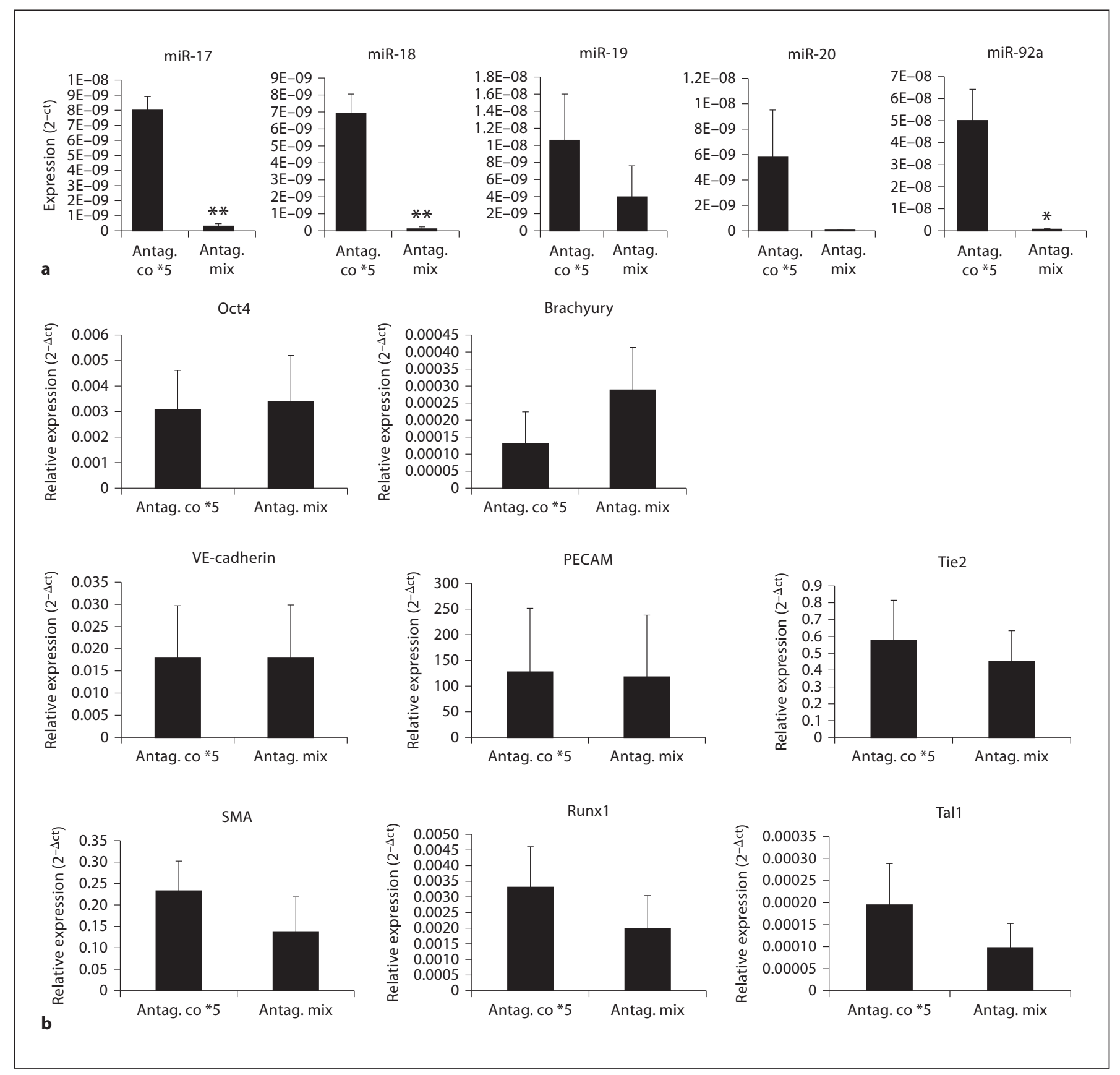

Fig. 8. A combination of antagomirs directed against the miR17-92 cluster does not affect endothelial differentiation. The experiment was carried out as described in figures 5 and 6 (VEGF protocol). The expression on day 7 is shown. a Antagomir-mixture included $150 \mathrm{nM}$ of each antagomir directed against miR-17, miR-18, miR-19, miR-20 and miR-92a. As a control, a higher concentration of antagomir-control (750 nM) was used. The data are depicted unnormalized. $\mathrm{n}=3-4 .{ }^{*} \mathrm{p}<0.05$. $\mathbf{b}$ The expression of pluripotency and endothelial marker genes was not altered by antagomir treatment. The data are presented as relative expression normalized to $\beta$-actin (Oct4, Brachyury, VE-Cadherin, SMA, Tal1 and Runx1) or PolR2A (Tie2, PECAM). n = 3-4. Antag. = Antagomir; $\mathrm{co}=$ control; $\operatorname{mix}=$ mixture. 


\section{Discussion}

This study demonstrates that members of the miR-1792 cluster are differentially expressed during the endothelial differentiation of both mESCs and miPSCs. While miR-17, miR-18 and miR-19 were increased upon induction of differentiation, miR-92a and the primary miR-1792 transcript were decreased. The observed upregulation of miR-17, miR-18, and miR-19 and the downregulation of miR-92a were consistent with previous data using an $\mathrm{miR}$ array to detect the expression of miRs during endothelial differentiation of human ESCs [14]. However, miR-20 was downregulated in the miR array, whereas in our experiments it was increased. Thus, although the miR-17-92 cluster members are derived from one common primary transcript, the expression of the mature miRs differs during the induction of endothelial differentiation. The differential expression pattern is consistent with previous findings showing that after induction of hindlimb ischemia the individual members are regulated with a different kinetic [30]. Moreover, a recent study documents that the individual members are differentially regulated during embryonic development [31]. Interestingly, during embryonic development, miR-17, miR-18 and miR-19 were significantly downregulated in lungs, whereas miR-20 and miR-92a showed a transient upregulation [31]. Together with our data, this study implicates that the members of the miR-17-92 cluster are posttranscriptionally regulated and may have different biological functions. Of note, several recent studies demonstrate that the posttranscriptional control of miRs plays an important role in regulating the levels of mature miRs at the level of Drosha or Dicer processing $[32,33]$.

Although the miR-17-92 cluster was shown to play a crucial role in endothelial cell biology and regulates sprouting angiogenesis, proliferation and neovascularization, the individual members of this cluster appear to not be essential for endothelial differentiation, despite an effective inhibition of the targeted miR by the antagomir treatment. However, inhibiting miR-92a specifically re- duced the hematopoietic markers Tal1 and Runx1. Since no effect of miR-92a inhibition on endothelial marker expression was detectable, we speculate that miR-92a might be required for appropriate hematopoietic development, a finding that is consistent with a recent study [34]. Moreover, the cluster inhibition induced a modest downregulation of the smooth muscle marker SMA, confirmed by a recent study [35]. Altogether, these results strongly suggest a role for miR-17-92 cluster during hematopoietic and smooth muscle cell differentiation rather than during endothelial differentiation.

Our data demonstrating that the inhibition of the members of the miR-17-92 cluster does not affect the expression of the commonly used endothelial markers during ESC differentiation - even though they have an important function in mature endothelial cells - is reminiscent of the endothelial-enriched miR-126, which is essential for endothelial cell functions, but also dispensable in endothelial differentiation [15].

To confirm that the lack of effects seen with the single antagomirs is not due to the compensatory upregulation of the related cluster members, we efficiently blocked the expression of all the mature miRs of the cluster by combining the antagomirs. However, the combined inhibition did not significantly affect endothelial cell differentiation. Of note, we cannot exclude that other miRs are upregulated to compensate for the loss of the miR-17-92 cluster. However, no obvious vascular defects were reported in the miR-17-92 knockout mice either [36]. These data indicate that the members of the miR-17-92 cluster may preferentially regulate endothelial cell functions under pathophysiological conditions.

\section{Acknowledgements}

The authors thank Marion Muhly-Reinholz for help with the immunostainings. This work was supported by the European Research Council (advanced grant 'Angiomir'; S.D.) and SFB834 (DFG). E.-M.H. is supported by a fellowship of the GRK1172 (DFG).

\section{References}

1 Wang S, Olson EN: Angiomirs - key regulators of angiogenesis. Curr Opin Genet Dev 2009;19:205-211.

- 2 Bonauer A, Boon RA, Dimmeler S: Vascular micrornas. Current Drug Targets 2010;11: 943-949.

3 Bonauer A, Dimmeler S: The microRNA17-92 cluster: still a miracle? Cell Cycle 2009; 8:3866-3873. $\checkmark 4$ He L, Thomson JM, Hemann MT, Hernando-Monge E, Mu D, Goodson S, Powers S, Cordon-Cardo C, Lowe SW, Hannon GJ, Hammond SM: A microRNA polycistron as a potential human oncogene. Nature 2005; 435:828-833.

5 Mendell JT: Miriad roles for the miR-17-92 cluster in development and disease. Cell 2008;133:217-222. 
-6 Ota A, Tagawa H, Karnan S, Tsuzuki S, Karpas A, Kira S, Yoshida Y, Seto M: Identification and characterization of a novel gene, c13orf25, as a target for 13q31-q32 amplification in malignant lymphoma. Cancer Research 2004;64:3087-3095.

$>7$ Dews M, Homayouni A, Yu D, Murphy D, Sevignani C, Wentzel E, Furth EE, Lee WM, Enders GH, Mendell JT, Thomas-Tikhonenko A: Augmentation of tumor angiogenesis by a Myc-activated microRNA cluster. Nat Genet 2006;38:1060-1065.

-8 Bonauer A, Carmona G, Iwasaki M, Mione M, Koyanagi M, Fischer A, Burchfield J, Fox $\mathrm{H}$, Doebele C, Ohtani K, Chavakis E, Potente M, Tjwa M, Urbich C, Zeiher AM, Dimmeler S: MicroRNA-92a controls angiogenesis and functional recovery of ischemic tissues in mice. Science 2009;324:1710-1713.

$>9$ Doebele C, Bonauer A, Fischer A, Scholz A, Reiss Y, Urbich C, Hofmann WK, Zeiher AM, Dimmeler S: Members of the microRNA-17-92 cluster exhibit a cell-intrinsic antiangiogenic function in endothelial cells. Blood 2010;115:4944-4950.

-10 Suarez Y, Fernandez-Hernando C, Yu J, Gerber SA, Harrison KD, Pober JS, Iruela-Arispe ML, Merkenschlager M, Sessa WC: Dicerdependent endothelial microRNAs are necessary for postnatal angiogenesis. Proc Natl Acad Sci USA 2008;105:14082-14087.

$\checkmark 11$ Kalka C, Baumgartner I: Gene and stem cell therapy in peripheral arterial occlusive disease. Vasc Med 2008;13:157-172.

12 Urbich: Relevance of monocytic features for neovascularization capacity of circulating endothelial progenitor cells. Circulation 2005;111:1718-1718.

13 Ohtani K, Dimmeler S: Control of cardiovascular differentiation by microRNAs. Basic Res Cardiol 2011;106:5-11.

14 Kane NM, Meloni M, Spencer HL, Craig MA, Strehl R, Milligan G, Houslay MD, Mountford JC, Emanueli C, Baker AH: Derivation of endothelial cells from human embryonic stem cells by directed differentiation: analysis of microRNA and angiogenesis in vitro and in vivo. Arterioscler Thromb Vasc Biol 2010;30:1389-1397.

15 Fish JE, Santoro MM, Morton SU, Yu S, Yeh RF, Wythe JD, Ivey KN, Bruneau BG, Stainier DY, Srivastava D: miR-126 regulates angiogenic signaling and vascular integrity. Dev Cell 2008;15:272-284.
16 Stadler B, Ivanovska I, Mehta K, Song S, Nelson A, Tan Y, Mathieu J, Darby C, Blau CA, Ware C, Peters G, Miller DG, Shen L, Cleary MA, Ruohola-Baker H: Characterization of microRNAs involved in embryonic stem cell states. Stem Cells Dev 2010;19:935-950.

17 Takahashi K, Yamanaka S: Induction of pluripotent stem cells from mouse embryonic and adult fibroblast cultures by defined factors. Cell 2006;126:663-676.

18 Feraud O, Cao Y, Vittet D: Embryonic stem cell-derived embryoid bodies development in collagen gels recapitulates sprouting angiogenesis. Lab Invest 2001;81:1669-1681.

19 Wang R, Clark R, Bautch VL: Embryonic stem cell-derived cystic embryoid bodies form vascular channels: an in vitro model of blood vessel development. Development 1992;114:303-316.

20 Korff T, Augustin HG: Integration of endothelial cells in multicellular spheroids prevents apoptosis and induces differentiation. J Cell Biol 1998;143:1341-1352.

21 Kaluza D, Kroll J, Gesierich S, Yao TP, Boon RA, Hergenreider E, Tjwa M, Rossig L, Seto E, Augustin HG, Zeiher AM, Dimmeler S, Urbich C: Class IIb HDAC6 regulates endothelial cell migration and angiogenesis by deacetylation of cortactin. EMBO J 2011;30: 4142-4156.

22 Vittet D, Prandini MH, Berthier R, Schweitzer A, Martin-Sisteron H, Uzan G, Dejana E: Embryonic stem cells differentiate in vitro to endothelial cells through successive maturation steps. Blood 1996;88:34243431.

23 Rosa A, Spagnoli FM, Brivanlou AH: The miR-430/427/302 family controls mesendodermal fate specification via species-specific target selection. Dev Cell 2009;16:517-527.

-24 Wang S, Aurora AB, Johnson BA, Qi X, McAnally J, Hill JA, Richardson JA, BasselDuby R, Olson EN: The endothelial-specific microRNA miR-126 governs vascular integrity and angiogenesis. Dev Cell 2008;15:261271.

25 Kim S, von Recum HA: Endothelial progenitor populations in differentiating embryonic stem cells I: identification and differentiation kinetics. Tissue Eng Part A 2009;15: 3709-3718.
6 Xiong JW: Molecular and developmental biology of the hemangioblast. Dev Dyn 2008; 237:1218-1231.

-27 Jezierski A, Swedani A, Wang L: Development of hematopoietic and endothelial cells from human embryonic stem cells: lessons from the studies using mouse as a model. Sci World J 2007;7:1950-1964.

28 Shivdasani RA, Mayer EL, Orkin SH: Absence of blood formation in mice lacking the T-cell leukaemia oncoprotein tal-1/SCL. Nature 1995;373:432-434.

29 Robb L, Begley CG: The SCL/TAL1 gene: roles in normal and malignant haematopoiesis. BioEssays 1997;19:607-613.

30 Bonauer A, Fischer A, Urbich C, Zeiher AM, Dimmeler S: Evidence for posttranscriptional control of individual members of the miR17-92 cluster. Circulation 2009;120:S1127.

31 Jevnaker AM, Khuu C, Kjole E, Bryne M, Osmundsen $\mathrm{H}$ : Expression of members of the miRNA17-92 cluster during development and in carcinogenesis. J Cell Physiol 2011;226:2257-2266.

32 Heo I, Joo C, Kim YK, Ha M, Yoon MJ, Cho J, Yeom KH, Han J, Kim VN: Tut4 in concert with lin28 suppresses microRNA biogenesis through pre-microRNA uridylation. Cell 2009; 138:696-708.

-33 Siomi H, Siomi MC: Posttranscriptional regulation of microRNA biogenesis in animals. Mol Cell 2010;38:323-332.

34 Li Y, Vecchiarelli-Federico LM, Li YJ, Egan SE, Spaner D, Hough MR, Ben-David Y: The miR-17-92 cluster expands multipotent hematopoietic progenitors while imbalanced expression of its individual oncogenic miRNAs promotes leukemia in mice. Blood 2012;119:4486-4498.

35 Chen Z, Wu J, Yang C, Fan P, Balazs L, Jiao Y, Lu M, Gu W, Li C, Pfeffer LM, Tigyi G, Yue $\mathrm{J}$ : DiGeorge syndrome critical region 8(DGCR8)-mediated miRNA biogenesis is essential for vascular smooth muscle cell development in mice. J Biol Chem 2012;287: 19018-19028.

$36 \mathrm{Mu}$ P, Han YC, Betel D, Yao E, Squatrito M, Ogrodowski P, de Stanchina E, D’Andrea A, Sander C, Ventura A: Genetic dissection of the miR-17-92 cluster of microRNAs in Mycinduced B-cell lymphomas. Genes Dev 2009; 23:2806-2811. 